\title{
Radar Detection of Moving Targets Behind Corners
}

\author{
Ain Sume, Magnus Gustafsson, Magnus Herberthson, Anna Jänis, \\ Stefan Nilsson, Jonas Rahm and Anders Örbom
}

\section{Linköping University Post Print}

N.B.: When citing this work, cite the original article.

(C2011 IEEE. Personal use of this material is permitted. However, permission to reprint/republish this material for advertising or promotional purposes or for creating new collective works for resale or redistribution to servers or lists, or to reuse any copyrighted component of this work in other works must be obtained from the IEEE.

Ain Sume, Magnus Gustafsson, Magnus Herberthson, Anna Jänis, Stefan Nilsson, Jonas Rahm and Anders Örbom, Radar Detection of Moving Targets Behind Corners, 2011, IEEE TRANSACTIONS ON GEOSCIENCE AND REMOTE SENSING, (49), 6, 2259-2267. http://dx.doi.org/10.1109/TGRS.2010.2096471 Postprint available at: Linköping University Electronic Press http://urn.kb.se/resolve?urn=urn:nbn:se:liu:diva-68912 


\title{
Radar Detection of Moving Targets Behind Corners
}

\author{
Ain Sume, Member, IEEE, Magnus Gustafsson, Magnus Herberthson, Anna Jänis, \\ Stefan Nilsson, Jonas Rahm, and Anders Örbom
}

\begin{abstract}
Detection of moving objects concealed behind a concrete wall corner has been demonstrated, using Doppler-based techniques with a stepped-frequency radar centered at $10 \mathrm{GHz}$, in a reduced-scale model of a street scenario. Micro-Doppler signatures have been traced in the return from a human target, both for walking and for breathing. Separate material measurements of the reflection and transmission of the concrete in the wall have showed that wall reflections are the dominating wave propagation mechanism for producing target detections, while wave components transmitted through the walls could be neglected. Weaker detections have been made of target returns via diffraction in the wall corner. A simple and fast algorithm for the detection and generation of detection tracks in down range has been developed, based on moving target indication technique.
\end{abstract}

Index Terms-Around corner, micro-Doppler, object detection, radar measurements, wall material.

\section{INTRODUCTION}

C OVERT information collection with surveillance sensors is a valuable ability in urban environment, e.g., in military operations or in related civil security engagements. Radar sensors provide an example of this, as they have potential for "seeing" around corners using the reflection, diffraction, and transmission of electromagnetic waves, both indoors and outdoors. Considerable research efforts have been directed toward the related application of observation through the walls, e.g., [1]-[12]. However, this is not the case in the application of interest here, which induced the Swedish Defence Research Agency (FOI) in 2006 to initiate a project entitled "Radar for detection of human activities around corners." The work in the project proceeds in steps with an increasing degree of realism of the scenario, including targets, as the main change between the steps. The emphasis is on radar measurements, but some supporting simulations and separate material investigations are being made in the progression. The radar investigations in each step consist of sample measurements, performed to give an indication if the see-around-corner principle can be expected to work well enough to warrant a progression to the next step. A possible application-oriented radar system design for a final

Manuscript received January 14, 2010; revised July 14, 2010 and September 3, 2010; accepted October 31, 2010. Date of publication January 5, 2011; date of current version May 20, 2011.

A. Sume, M. Gustafsson, A. Jänis, S. Nilsson, J. Rahm, and A. Örbom are with the Information Systems, Swedish Defence Research Agency, 58111 Linköping, Sweden (e-mail: ain.sume@foi.se; magnus.gustafsson@foi.se; anna.janis@foi.se; stefan.nilsson@foi.se; jonas.rahm@foi.se; anders.orbom@ foi.se).

M. Herberthson is with the Swedish Defence Research Agency, 58111 Linköping, Sweden, and also with the Linköping University, 58183 Linköping, Sweden (e-mail: magnus.herberthson@foi.se).

Color versions of one or more of the figures in this paper are available online at http://ieeexplore.ieee.org.

Digital Object Identifier 10.1109/TGRS.2010.2096471 realistic environment will require a fuller variation of parameters and detailed electromagnetic field simulations. We started in 2007 with a stylized model setting with metallic walls and target reflectors of simple shape in a well-controlled movement and have proceeded to concrete walls and a human target.

The radar findings of the mentioned previous work [13]-[16], made at X-band frequencies centered at $10 \mathrm{GHz}$, demonstrated the capacity of Doppler techniques to detect moving objects consisting of radar reflectors and a human target, with no direct line of sight (LOS) between the objects and the radar. To our knowledge, these findings are the first published ones demonstrating the potentiality of this application.

Since there is no LOS in our application, the target return arrives at the radar via indirect paths involving reflection, diffraction, and possibly refraction. In a varied environment, the wave propagation from the target will reach the radar along many paths. Thus, multipath propagation is a central topic for analysis in connection with detection and tracking. Some results that are relevant to our work can be found in other related applications in urban surveillance in the literature. For instance, in [17], a quasi-analytical model for non-LOS (NLOS) propagation assuming multiple specular reflections in buildings is derived to evaluate radar coverage from an airborne radar platform. The model predicts that NLOS could offer a significant improvement in coverage rate. The same application-airborne localization and tracking of vehicles within urban canyons utilizing multipath propagation between buildings-is being studied in Defense Advanced Research Projects Agency's Multipath Exploitation Radar program [18], with published results from simulations [19] and from supporting ground-based measurements [20]. In the latter work, the electromagnetic phenomenology was studied at X-band, including field strengths and spatial coherency of the energy reflected from building walls.

In the through-the-wall application, the potential use of multipath returns for detection and tracking has been analyzed in [21]. In this latter application, various clutter cancellation techniques have been applied for observing human targets with some movement, e.g., [9], [22], and [23]. The techniques are based on forming some difference between subsequent recordings of a target scene, in order to eliminate nonmoving clutter [moving target indicator (MTI) filtering]. In [22], this difference is made of subsequent returns of monochromatic transmissions, whereupon a fast fourier transform (FFT) of the cleaned data displays the breathing spectrum, however, with no range discrimination. In [23], where the waveform is an ultrawideband impulse, two methods for forming the difference are used to implement an MTI filter: For human micromotion 


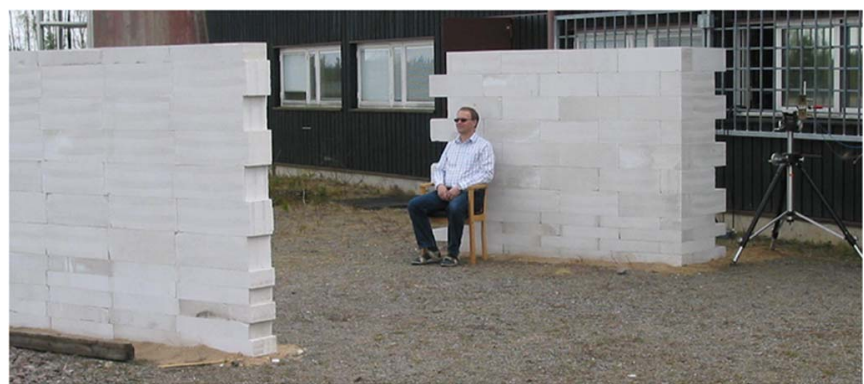

Fig. 1. Setup of (right) radar antennas, a human target, and concrete wall sections.

detection, the average of all received pulse echoes is subtracted from the currently received one, whereas for macromotion, a pulse canceller is used [24].

Our present paper is a continuation of the mentioned FOI work, presenting further processing of the data of the human target, which has resulted in the detection of breathing, with a characteristic Doppler signature. Also, a simple algorithm for fast detection and track generation in down range of a moving object behind the corner is introduced. The approach is basically that used in the through-the-wall case of [9], applied with some additions to reflector measurement data in our multiwall scenario. We have also made separate new measurements of the reflection properties of building materials, including the light concrete block material used for the walls in the test-range setup of a corner model.

\section{EQUiPMENT}

A short description of the equipment used for the radar measurements is given here. More details can be found in [15].

The measurements were conducted on the FOI test range. The arranged setup, shown in Fig. 1, was intended to mimic a street scenario on a reduced scale. It consisted of two perpendicular wall sections of light concrete forming a corner at the right, with an opposite wall at the left.

The light concrete building blocks which formed the walls, each measured $20 \times 20 \times 60 \mathrm{~cm}^{3}$ (width $\times$ height $\times$ length) and weighed $16 \mathrm{~kg}$. The blocks were massive with no hollows. Some blocks were of half height, i.e., $10 \mathrm{~cm}$. The overall dimensions of the wall sections are given in Fig. 2. The distance between the two parallell wall sections was $4.88 \mathrm{~m}$.

Two identical standard gain horns with $30^{\circ} \times 30^{\circ} \boldsymbol{E} \times$ $\boldsymbol{H}$-plane half-power lobes were used for transmitting and receiving antennas, respectively, stacked vertically on a tripod (at the far right in Fig. 1), with horizontally adjusted axes. The transmitting horn was placed with its axis $12 \mathrm{~cm}$ above that of the receiving horn and $1.40 \mathrm{~m}$ above the ground. In azimuth, they were either directed obliquely toward a point on the opposite wall for propagation through reflection, or they were directed toward the wall corner for diffraction around it. Horizontal polarization ( $\boldsymbol{E}$ vector parallel to the ground) was chosen throughout. This choice was made with consideration to the higher around-corner diffracted energy that it would give compared to the orthogonal polarization, based on field calculations, [25], [26], made for the first series of measurements

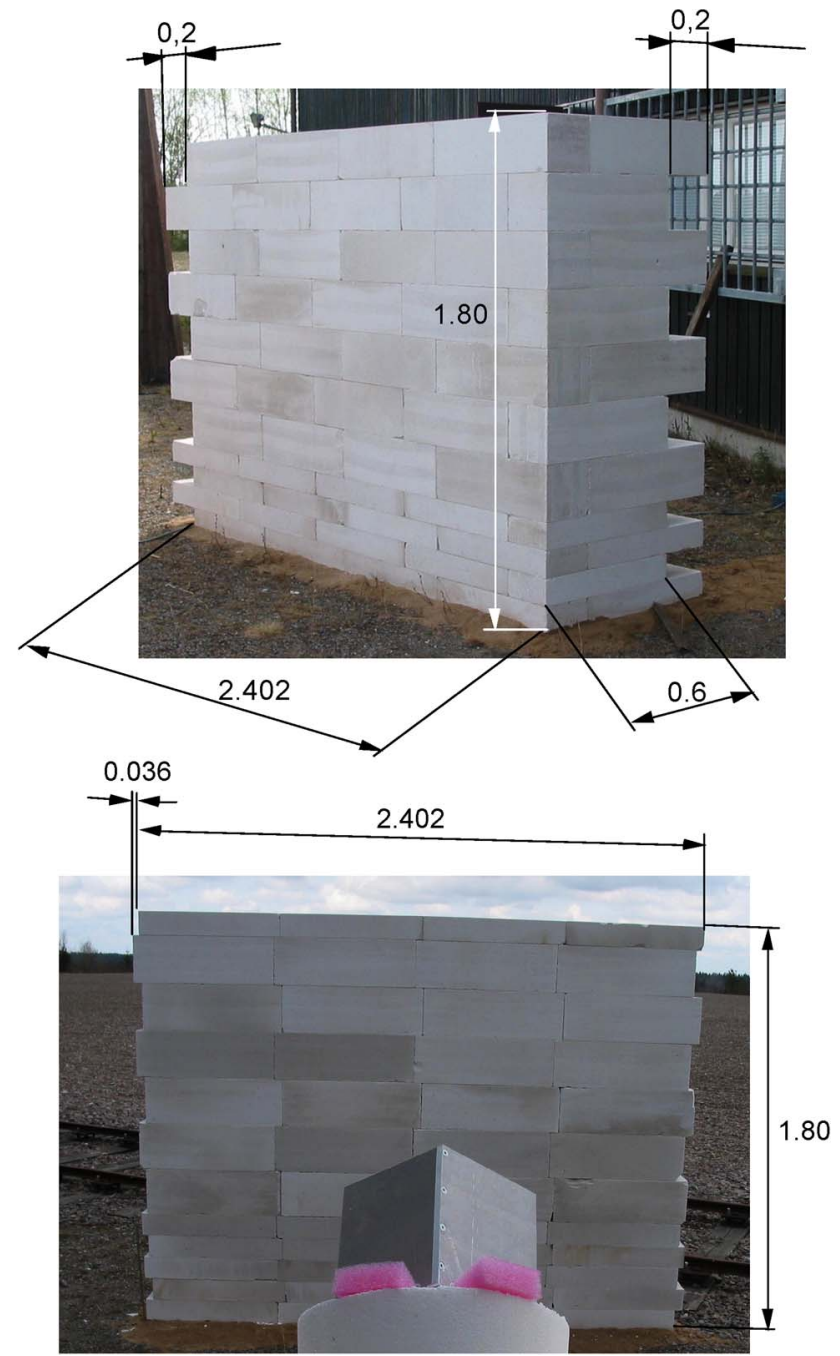

Fig. 2. Dimensions (m) of the two wall sections. The back side of a calibration reflector appears in the foreground of the second figure.

carried out in this project, with metallic walls. We have not determined the permittivity of our concrete wall material. If we use the published values of the real and imaginary parts of the relative permittivity, $\varepsilon^{\prime}=7.7$ and $\varepsilon^{\prime \prime}=0.33$, respectively, for concrete [27], at about $10 \mathrm{GHz}$, the field reflection coefficient becomes about $2 \%$ lower than for the orthogonal polarization [28], for a 20-cm-thick concrete sheet, at the value of the incidence angle in our measurements, i.e., $11^{\circ}$. This is well away from the Brewster angle occurring at about $70^{\circ}$ incidence angle [28], where the reflection coefficient for the polarization chosen here becomes very small. Generally, the optimal polarization choice will depend on the specific geometry of the setup.

The coherent radar cross-section measurement system of the test range was used, synthesizing the spectrum of a shortpulse transmitted waveform by frequency stepping of a gated continuous wave waveform centered around $10 \mathrm{GHz}$. The frequency stepping period was $10 \mu \mathrm{s}$. In the measurements of human movement, an interval of $B=108 \mathrm{MHz}$ was covered in $N=27$ steps of $4 \mathrm{MHz}$ each, which took $0.27 \mathrm{~ms}$. This was the largest processed bandwidth for the data presented here for human movement. Amplitude and phase were recorded on reception. 


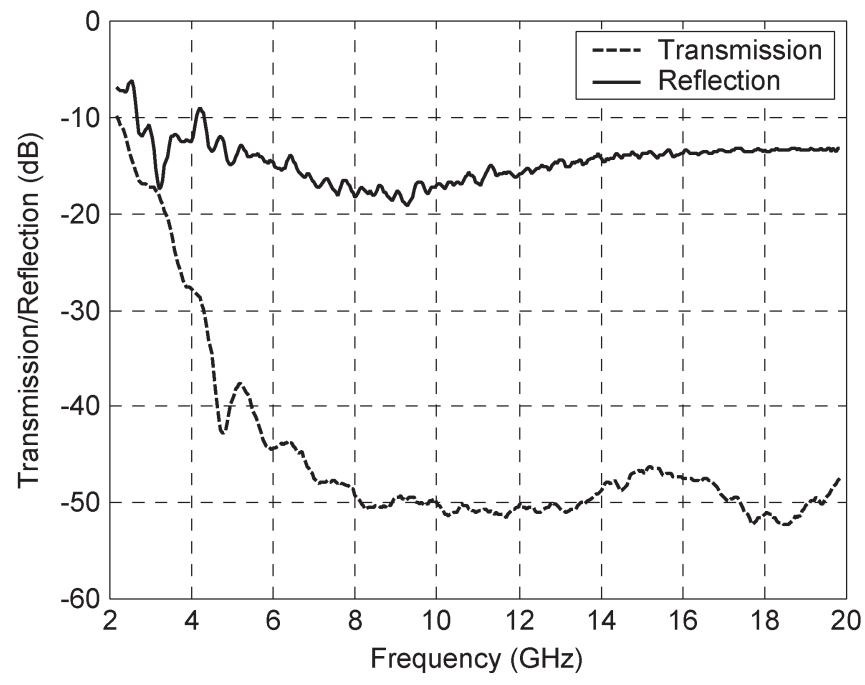

Fig. 3. Measured power transmission and reflection coefficients as functions of frequency of porous concrete block with a thickness of $20 \mathrm{~cm}$ and a moisture content of $23 \%$. Above $9 \mathrm{GHz}$, the transmission curve shown coincides with the (frequency dependent) noise floor.

\section{Material MeAsurement}

As part of a more extensive separate measurement program for characterization of building material [29], we have made free-space measurements of the transmission and reflection of the porous light concrete blocks, which were used for the wall material. The measurements, using network analyzers, were described in [29] and [30]. Briefly, the transmission was determined with the material sample placed in the space between a transmitting and a receiving horn antenna. In order to eliminate disturbing signals, like crosstalk between the antennas and interference with the surrounding, the space between the sample and the receiving horn was enclosed by a metallic box with its inner walls covered with radar absorbing material. Two opposite holes were cut in the walls. One hole served as an inlet and held the material sample, while the outlet hole enclosed the aperture of the receiving horn. In the reflection measurements, the transmitting and receiving antennas, as well as the material sample, were mounted on vertical axes, which enabled rotation of the sample and the receiving antenna, in order to vary the incidence angle. Fig. 3 shows the measured power transmission and reflection coefficients as functions of frequency, for normal incidence toward the façade-forming surface. These coefficients, $T$ and $R$, are defined as the square of the ratio of transmitted and reflected electric fields to the incident field, respectively, as measured by the network analyzer. The moisture content by weight of the measured block was $23 \%$, the same as for the blocks in the wall during the outdoor radar measurements. The moisture content at a specific point in time was determined from a comparison of the weight of a sample block at that time and the weight after drying in an oven.

As can be seen from the figure, the transmission is more than $30 \mathrm{~dB}$ below the reflection at $10 \mathrm{GHz}$. As mentioned, we did not determine the permittivity for the material and cannot compare this measured difference with an accurately calculated one. We can make a rough calculation using the mentioned published value for concrete, $\varepsilon^{\prime}=7.7, \varepsilon^{\prime \prime}=0.33$, at about $10 \mathrm{GHz}$ and a water-to-cement ratio of 0.5 [27]. This gives a value of $-23.8 \mathrm{~dB}$ and $-6.5 \mathrm{~dB}$ for the power transmission and reflection coefficients, respectively, using standard theory for a 20 -cm-thick conducting infinite sheet in air at normal incidence [31]. Higher values of the imaginary part of the permittivity would give proportionally lower decibel-transmission values. Differences in concrete type and physical conditions contribute to the discrepancy between our measured values and the calculated ones. Without knowledge of the exact composition of different investigated specimens, it is difficult to account more precisely for differences in transmission and reflection properties. As an additional comparison, the measurements made by the US National Institute of Standards and Technology of concrete blocks of the same thickness as ours $(20 \mathrm{~cm})$ gave transmission coefficients roughly in the -60 to $-70 \mathrm{~dB}$ range at $8 \mathrm{GHz}$ [32]. However, their density was about 3.5 times higher than that of our blocks, indicating that the concrete was not of our lightweight type. Our material measurements allow us to draw the general conclusion of relevance to the radar measurements that the wave components which penetrate the wall along a direct pathway to the target and back to the radar are very much attenuated and can be neglected compared to the received returns from components propagated through free space, around the corner through reflections in the walls. The measurements have confirmed this conclusion insofar that we have seen no target return that can be ascribed to throughwall propagation. In an indoor situation, where one can expect more transparent wall materials, through-corner propagation may become significant.

\section{Signal PRocessing}

Three signal processing methods were used for radar measurements. The initial analysis, aimed at detection of the reflector targets in uniform motion, was made using standard Doppler signal processing, including a 2-D FFT processing, e.g., [15], [33]. The first transform, an inverse FFT, takes the $N$ samples of each frequency sweep of bandwidth $B$ into a range profile, while the second FFT transforms the samples of the same range cell ("gate") from consecutive frequency sweeps to provide Doppler information. The detection is then made in a specific gate, guided by good knowledge of the location of the reflector target as a function of time. The range resolution is given by $c /(2 B)$ [24], where $c$ is the velocity of light.

A variant of the described method is used for the second choice. It is the short-time fourier transform (STFT), intended to follow the time development of the Doppler shift of a target moving with nonuniform speed [34]. For this, we have used the standard MATLAB spectrogram routine.

The third method, aimed at automatic fast detection and down-range track generation of objects, is an MTI algorithm. It will be described in more detail in Section VI.

\section{Measurements of Human Doppler}

\section{A. Walking}

Measurements of the radar signatures of two forms of human movement are reported here, viz., walking and breathing. The 


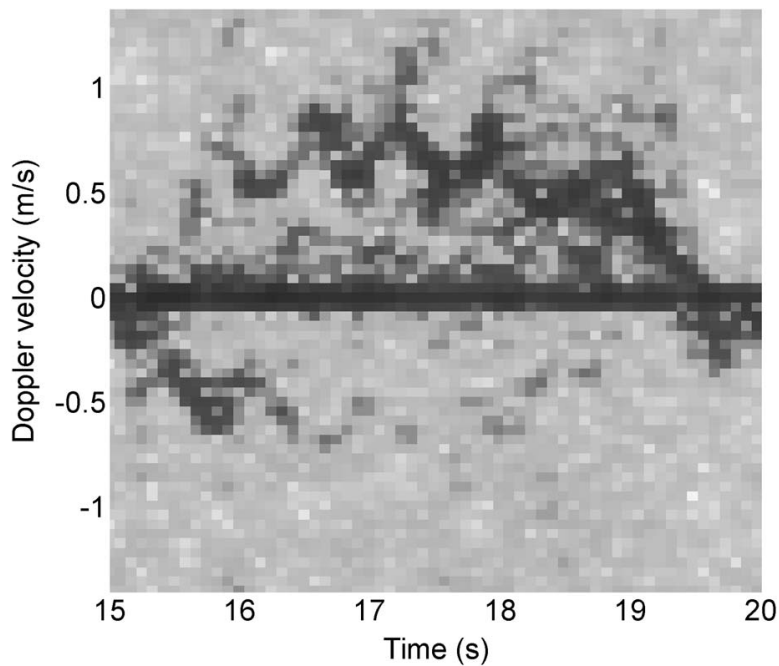

Fig. 4. Time/velocity diagram of the walk from corner B to A in Fig. 5. An increased tone of black corresponds to an increased signal level.

person walked along a rectangular path in the region between the two parallel wall sections in Fig. 1. Some results were described in [15] together with the comparison of the model signature of [35]. Fig. 4 is a further example with improved grayscale representation of the two dominating "micro-Doppler" signatures appearing in a specific range gate in the radar shadow zone behind the corner.

Fig. 4 has been generated using the standard MATLAB spectrogram routine involving sliding 64-point STFTs, corresponding to 0.3 -s interval with $75 \%$ overlap and Hamming weighting. The range resolution is $2.2 \mathrm{~m}$, given by the width of the processed frequency interval. The tentative identification made in [15] of the signature was that it consists of returns from the torso and the legs, respectively.

One may note the simultaneous appearance of two time/ velocity tracks in Fig. 4, one stronger at positive and one weaker at negative velocities, respectively, being mirror images of each other. This could be interpreted as two wave components arriving in the same gate, having propagated different paths whose lengths differ by less than the range gate depth of $2.2 \mathrm{~m}$. Figs. 5 and 6 show two possibilities, which are consistent with this requirement and with the opposite Doppler signs. One notes a gradual increase in intensity with time for the upper signature track in Fig. 4 as opposed to a decrease for the lower track. One possible qualitative interpretation is that this is a consequence of two influences on the radar return. The first is a dependence of the target return on the synthesized pulse shape in range. The second, only relevant for the pathway in Fig. 6 which involves diffraction in the corner, is a strong dependence on the angle $\beta$, a measure of the target's advancement into the shadow region. The mentioned dependences work as follows: About the shown position of the target in Figs. 5 and 6, the propagation lengths along the antenna beam center paths are equal for the two respective pathways. At this point, the target is located at the beginning of the considered range gate, i.e., in the short-range part. When the target moves from this point toward the corner A of the rectangular path, the radar return shown in Fig. 5 shifts into the range gate toward longer ranges, whereas the return

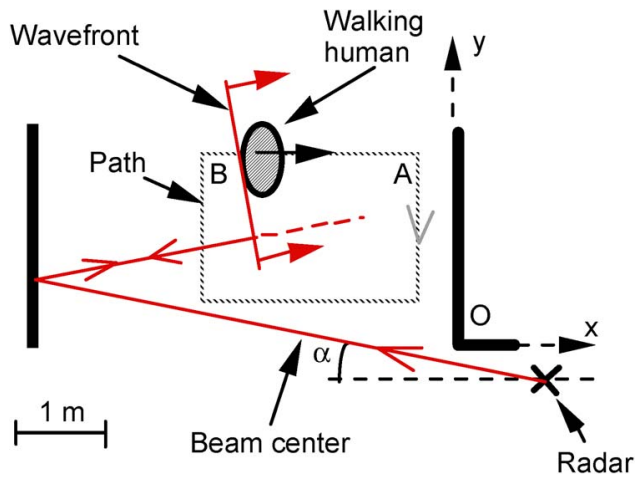

Fig. 5. Sketch of the measurement scene, with human target walking round a rectangular path of size $2.5 \times 1.7 \mathrm{~m}$. The corner A is located at $x=-0.4 \mathrm{~m}$, $y=2.05 \mathrm{~m}$, the radar at $x=1.05 \mathrm{~m}, y=-0.50 \mathrm{~m}$, in an $x, y$-system with origin at $\mathrm{O}$, defined as the intersection between the ground plane and the "street" side surfaces of the walls forming the corner. The horizontal axes of the antennas form an azimuth angle $\alpha=10.7^{\circ}$ with the $x$-axis. The indicated beam center ray is shown with specular reflection in the wall at the left. The interaction of the reflected beam with the target occurs off the beam center and is represented in a stylized form via a plane wave front associated with the beam. The velocity diagram for the walk from B to A is shown in Fig. 4 by the upper stronger signature track at positive velocities.

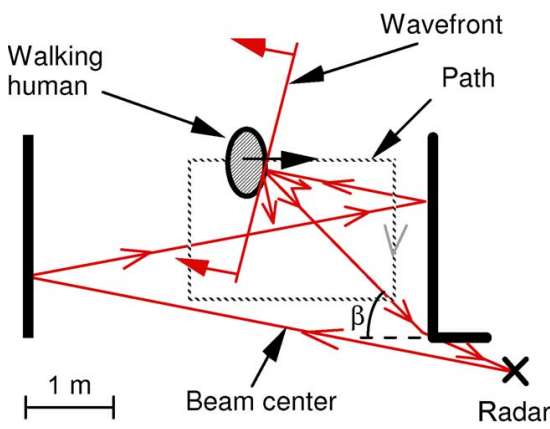

Fig. 6. Same as Fig. 5 but with ray pathway candidate for the negative velocity track in Fig. 4.

shown in Fig. 6 moves in the opposite direction, out of the gate. This would give the intensity development shown in Fig. 4. An additional contribution to this development for the case in Fig. 6 is provided by the strong decrease of the radar return via diffraction in the corner as a function of the increased angle $\beta$ [25], [26]. This contribution is the major one. The given arguments should be seen as presenting possibilities in agreement with the data. Stronger claims would require support from quantitative field calculations, which are beyond the scope of this paper.

We have discussed two dominant pathways consistent with a specific measurement. Generally, even in this relatively "clean" environment, the multipath situation is quite complex, with both monostatic and bistatic propagations, including reflection and diffraction. The interpretation of data in this situation needs careful treatment. The illumination of the scene from several aspects, shown in Figs. 5 and 6, could possibly be taken advantage of, since it contains information for target location, for example. We will discuss this topic further in Section VI.

\section{B. Breathing}

We have detected human breathing of a person sitting on a chair, deep in the shadow region behind the corner as shown in 


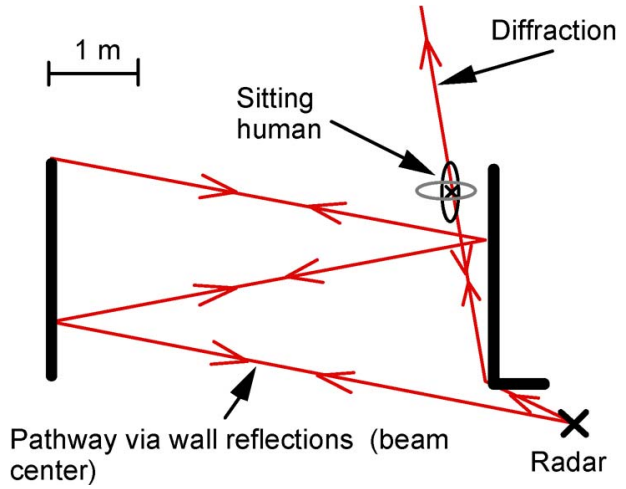

Fig. 7. Sketch of the two major wave pathways, with sitting human target. The target has been placed at the far end of the wall around the corner, while the position and orientation of the radar antennas are unchanged from Figs. 5 and 6. The target is then located off the reflected beam center and does not present the easiest detection case.

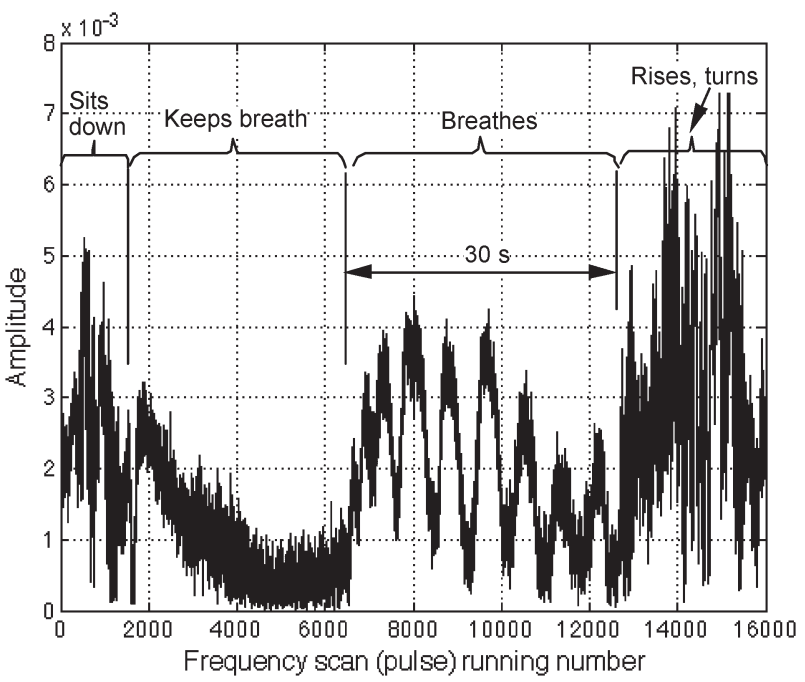

Fig. 8. Amplitude as a function of frequency sweep number (proportional to time) of radar return from the sitting human, facing the opposite wall, with annotated activities. The chosen range gate corresponds to the traveled distance of the wave component reflected from the walls in Fig. 7. Stationary background has been subtracted as described in the text.

Fig. 1. The detection is unquestionable for the wave component propagating to the target and back to the radar via a reflection in the opposite wall, as shown in Fig. 7.

It turned out that the STFT signal processing scheme used for the analysis of walking did not produce the clearest detection. The integration intervals will be too short in this case, and the low speed of the body parts participating in the movement is not distinctly separated from the returns from stationary background (zero-Doppler). Instead, the movement emerges clearly in the time functions of the amplitude and the phase of the signal in the range gate containing the human target, with a background subtraction to eliminate returns from stationary objects in the gate. Since no special background measurement was made on the scene without target, we instead used the target recording during approximately $15 \mathrm{~s}$ without breathing, preceding a $30-\mathrm{s}$ interval with breathing. The mean value of the complex signal during the $15 \mathrm{~s}$ was taken to represent stationary "background" and was subtracted from the target recording in the same range gate. Fig. 8 shows the resulting amplitude curve

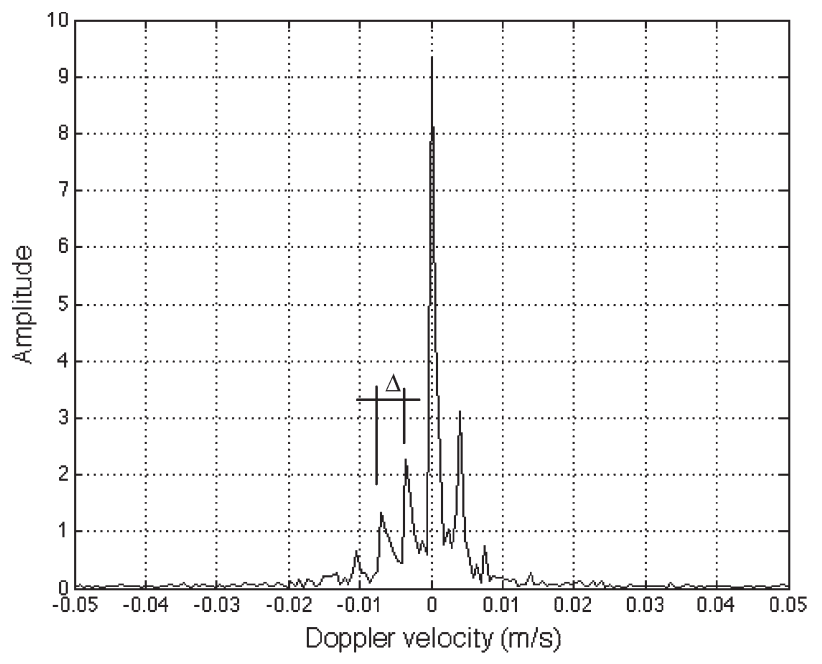

Fig. 9. Doppler velocity spectrum of breathing in Fig. 8, showing the main components at $\pm 0.0036 \mathrm{~m} / \mathrm{s}$ and the sidebands due to periodic phase modulation.

versus time, with annotations of the activities of the person. The pattern during the breathing has a period of about $4 \mathrm{~s}$, in accordance with the real movement.

Fig. 9 shows the result of a fourier transform (FT) (Doppler integration) of the complex signal from the person (with subtracted background) over the $30 \mathrm{~s}$ of breathing. The residual stationary background is seen in this velocity diagram at zero velocity. Furthermore, at least five spectral peaks are seen at both negative and positive velocities, with approximately equal separation $\Delta=0.0036 \mathrm{~m} / \mathrm{s}$ between two adjacent peaks. The pattern corresponds to the side-band pattern associated with an object with temporally sinusoidal vibrational movement along the radar propagation direction [36]. Modeling the breathing with such periodic movement of the form $\sin (\omega t+k \sin \Omega t)$, where $\omega$ and $\Omega$ are the angular frequencies of the incident wave and vibration, respectively, and $k$ is a modulation coefficient, the period of the movement can be calculated from the measured value of $\Delta$. If $T=2 \pi / \Omega$ is the period of the vibration, one has with $\lambda=3 \mathrm{~cm}$, that $T=\lambda /(2 \Delta)=4.1 \mathrm{~s}$, in agreement with the breathing of the test object. Hence, we conclude that the pattern is associated with the breathing and is not some form of interference. In addition to the pattern of side-band peaks, there is also a pedestal between approximately $\pm 0.015 \mathrm{~m} / \mathrm{s}$, probably also due to the breathing movement, which is not of the exact sinusoidal form assumed in the model but also contains other more irregular components.

The detection of breathing in the shown case (Fig. 8) can be made almost equally well without any background subtraction since regular breathing movement appears with approximately the same signal to noise ratio in the raw data signature (not shown) prior to subtraction. The subtraction produces in the shown case a smoother baseline. The procedure in the form applied here should be seen as an offline postprocessing method where one is not hard-pressed for time but can analyze the collected data to pick a suitable reference background. In a real dynamic situation, one has to resort to some faster automatic scheme. An example will be presented in Section VI in the form of an MTI-like Doppler detection algorithm. 


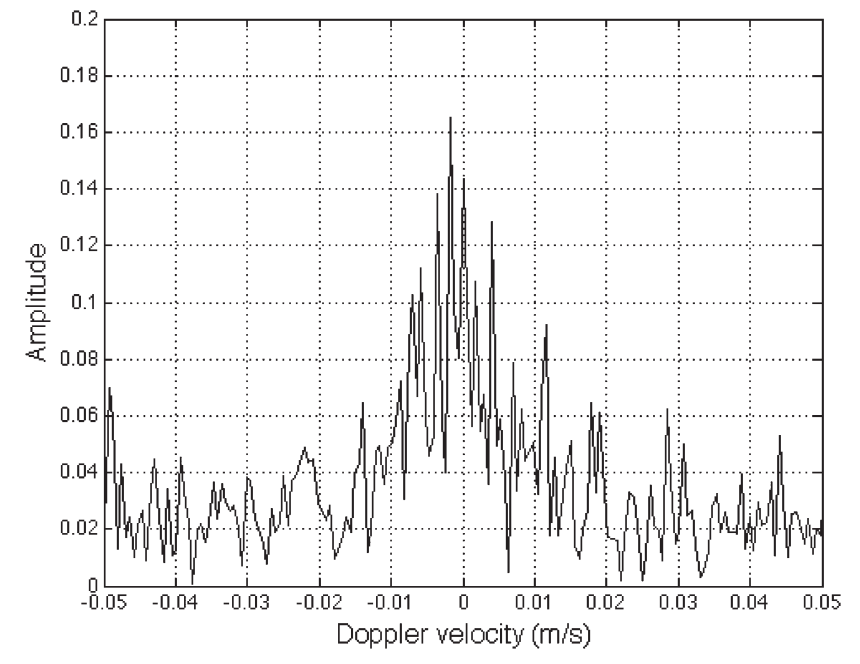

Fig. 10. Doppler velocity spectrum of the sitting breathing person as indicated in Fig. 7 but now facing the corner. The chosen range gate corresponds to the diffracted component.

There is also a probable detection of breathing from the other propagation path drawn in Fig. 7, which is the diffracted wave, in the case where the person is sitting facing the corner. The same signal processing as for the reflected wave just treated produces the Doppler spectrum shown in Fig. 10. The same broad pedestal is present between $\pm 0.015 \mathrm{~m} / \mathrm{s}$, as in the previous case, probably due to the breathing movement. A possible side-band pattern with peaks as in Fig. 9 is hidden in the general background fluctuations. The Doppler velocity spectrum is slightly asymmetric with respect to zero velocity. We believe it to be due to the fact that the backscatter from the human body is very sensitive to the exact geometry of the scattering surface, which is likely to differ slightly between inhalation and expiration, as is the chest speed. The sensitivity is demonstrated by the amplitude variation during the nonbreathing period in Fig. 8, which results from some very small movement of the upper body.

\section{MTI ALGORITHM FOR DETECTION AND DOWN-RANGE TRACK GENERATION}

The above-mentioned results indicate that it is feasible to detect moving objects in the shadow region behind corners, at least in favorable circumstances. The detection, however, is facilitated by the condition that the position of the object is known to a precision of a few wavelengths. No unbiased search has been conducted among data from the whole surveyed area. In a real scenario, the position of a target will seldom be known a priori. Hence, an important task is to find simple, fast, and reliable signal processing algorithms that can be used to extract in real time the signal return from a moving target in a shadow region. For this reason, as a first algorithm development, we have tested a relatively simple and fast MTI algorithm [9], [24].

The data used for the algorithm development were obtained from measurements of a metallic sphere, with a radius of $0.25 \mathrm{~m}$, mounted $0.7 \mathrm{~m}$ off center on a turntable, on a horizontal arm, producing a circular orbit with a measured period time of $584 \mathrm{~s}$, corresponding to an orbital speed of about $0.0075 \mathrm{~m} / \mathrm{s}$. There was no direct LOS between the antennas and the sphere. The antenna main lobes were directed toward the wall corner,

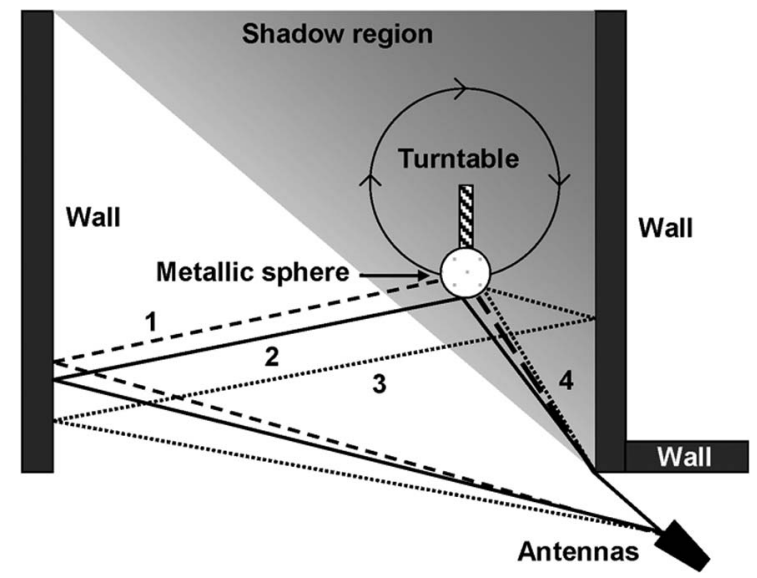

Fig. 11. Sketch of measurement setup and four possible ray paths in the measurements of a metallic sphere. (Short dashes) Path 1 involves bistatic reflection in the left wall and backscatter from the sphere. (Solid) Path 2 involves bistatic reflection in the left wall and the sphere and the diffraction in the corner. (Dotted) Path 3 involves bistatic reflections in the two parallel walls and in the sphere, as well as diffraction in the corner. (Long dashes) Path 4 involves diffraction in the corner and backscatter from the sphere.

but they also illuminated the wall on the opposite side cf. Fig. 11, which shows the setup with some possible ray paths indicated.

The frequency stepping in this case went from 8 to $12 \mathrm{GHz}$, in 1001 steps of $4 \mathrm{MHz}$ each. Considering the $10-\mu \mathrm{s}$ step time between two frequencies, the sampling rate for a complete frequency sweep synthesizing a short radar pulse with this set of parameters could be at most $\sim 100 \mathrm{~Hz}$. In the measurements, a sweep rate of $50 \mathrm{~Hz}$ was chosen.

The signal processing is based on coherent subtraction between different frequency sweeps. This method effectively reduces static background returns and produces a resulting signal originating from the phase shift of the moving object and a residual originating from phase noise. The phase shift difference between the two sweeps has to be sufficiently large to produce an appreciable resulting signal above the noise after the subtraction. A crucial parameter is, thus, the time distance between the two sweeps involved. The slow orbital motion of the target in this measurement $(0.0075 \mathrm{~m} / \mathrm{s})$ in combination with a high sweep sampling rate will cause small phase shifts if the subtraction is made of consecutive sweeps, which results in no detection. It is, therefore, essential to provide a facility for varying the time distance between the sweep samples in order to be able to detect different object velocities. The subtraction is done in the following manner

$$
\operatorname{Data}(f, \Delta T)=\operatorname{Data}\left(f, T_{p}\right)-\operatorname{Data}\left(f, T_{p+q}\right)
$$

where Data is a complex value, $p$ is the sweep number index, and $q$ is the index which represents the time distance between two subtracted sweeps. Table I is a 2-D matrix representation of the arrangement of the frequency/time data.

By using the inverse FFT (IFFT) on the resulting data, it is possible to make down-range plots and, from these, create movies which enable playback of the measurements. By including thresholds in the down-range movies, it is possible to get a plot of detection as a function of time, as can be seen in Fig. 12. 
TABLE I

ARRANGEMENT OF FREQUENCY/TIME DATA

\begin{tabular}{|c|c|c|c|c|c|c|}
\hline & $\mathrm{T}_{1}$ & $\mathrm{~T}_{2}$ & $\mathrm{~T}_{3}$ & $\mathrm{~T}_{4}$ & $\ldots$ & $\mathrm{T}_{\mathrm{N}}$ \\
\hline $\mathrm{f}_{1}$ & Data $_{11}$ & Data $_{12}$ & Data $_{13}$ & Data $_{14}$ & $\ldots$ & Data $_{1 \mathrm{~N}}$ \\
\hline $\mathrm{f}_{2}$ & Data $_{21}$ & Data $_{22}$ & Data $_{23}$ & Data $_{24}$ & $\ldots$ & Data $_{2 \mathrm{~N}}$ \\
\hline $\mathrm{f}_{3}$ & Data $_{31}$ & Data $_{32}$ & Data $_{33}$ & Data $_{34}$ & $\ldots$ & Data $_{3 \mathrm{~N}}$ \\
\hline$\ldots$ & $\ldots$ & $\ldots$ & $\ldots$ & $\ldots$ & $\ldots$ & $\ldots$ \\
\hline $\mathrm{f}_{\mathrm{n}}$ & Data $_{\mathrm{n} 1}$ & Data $_{\mathrm{n} 2}$ & Data $_{\mathrm{n} 3}$ & Data $_{\mathrm{n} 4}$ & $\ldots$ & Data $_{\mathrm{nN}}$ \\
\hline
\end{tabular}
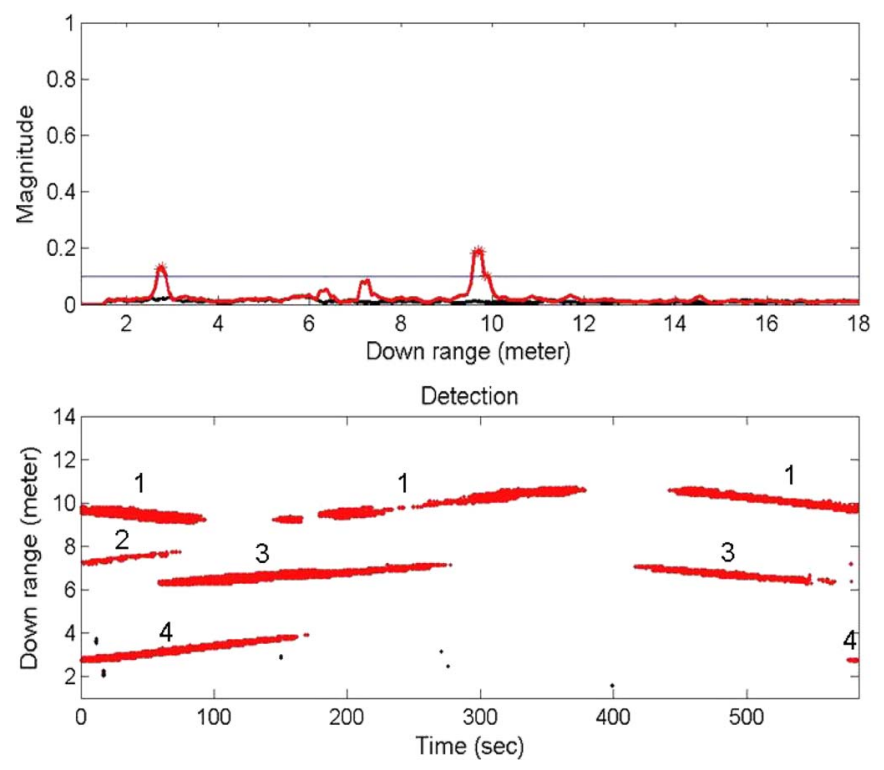

Fig. 12. (Upper) Down-range plot of radar return from metallic sphere in circular movement. The plot is the result of MTI filtering (subtraction) of two frequency sweeps separated by 20 sweep periods, followed by an IFFT and a smoothing procedure with a running average filter including two range bins. The MTI filtering is applied over the whole collection interval (583 s) with a forward translation of 20 sweeps between subsequent subtractions. The shown plot is the last one, produced at the end of the interval. A threshold for detection is indicated by the horizontal line at a magnitude of 0.1. (Lower) Declared detections versus time for all down-range plots. The numbers in the graph associate the tracks with the corresponding ray paths in Fig. 11.

For the smoothing of the down-range plots, a running average filter is used.

Fig. 12 shows that it is possible to detect a moving object in the shadow region around a corner, with no direct LOS to the antennas. The different tracks in the figure correspond to different propagation paths of the electromagnetic wave, as shown in Fig. 11 by the different lines, resulting in several peaks in the down-range plots. The long-dashed, short-dashed, solid, and the dotted paths give rise to the detection tracks at about 3 , 10,6 , and $7.5 \mathrm{~m}$, respectively (see Fig. 12). The sensitivity of this method depends on several parameters, e.g., the velocity of the moving object, the radar system parameters (output power and sweep-rate sampling), and the antenna properties (bandwidth limitations and lobe width). Also, the parameter settings of the offline analysis are influential, like the time distance between two subtracted sweeps, the threshold level in the detection, etc.

It should also be commented on the possibility to position targets via their detections. Since a certain target can be detected at several distances, corresponding to different propagation paths, this information can be combined and used to position the target. To accomplish such a positioning, the geometry of the scattering environment must be known. The actual position is then obtained through relatively straightforward ray tracing. Note, however, that the presence of several targets complicates this procedure noticeably since it is not clear a priori how to decide whether different detections correspond to the same target or not. This so-called association problem is addressed by using Doppler information and by following the detections over time.

Finally, a few remarks should be made whether the Doppler techniques described in this paper are generally applicable. They obviously fail if the probing field illuminating the target is too weak, or lacking altogether, or if the movement producing the Doppler shift is orthogonal to the incident field propagation. In the shadow region behind the corner, this incident field is determined by the superposition of the specularly reflected wave component and the diffracted one, the strength mainly dictated by the former component. Hence, regions of decreasing field strength can be expected away from the beam center of the specularly reflected component. The exact illumination can be quite complex due to the possible multitude of illumination cases cf. Figs. 5 and 6 and depends on the specific geometry. For the diffracted field, there is a rapid decrease of the field strength with increasing penetration into the shadow region (angle $\beta$ in Fig. 6). More specific quantitative statements need field calculations, which are beyond the scope of this mainly experimental paper. In principle, however, there is always some illumination of the target in our scenario due to the existing multitude of propagation paths. This circumstance also produces Doppler shifts irrespective of the direction of the movement. The strength is again dependent on the specific geometry and on the scattering properties of the target.

\section{CONCLUSION}

The investigations have demonstrated the possibility with $\mathrm{X}$-band radar to detect moving human objects concealed behind concrete wall corners. The detection was realized with steppedfrequency radar in a relatively controlled reduced-scale scenario using the return from the wave component that reaches the target via specular reflections in adequately positioned walls, or via diffraction in the corner itself, or via a combination of these pathways. Separate measurements of the reflection and transmission properties of the wall material have indicated low transmission through a corner formed by this wall type, leaving the reflected and diffracted wave components as dominant agents for the interaction with targets used for detection. The movements monitored here consist of human walking and breathing. Signal processing with standard 2-D FT, including the STFT, was used to produce time/velocity diagrams of human walking, including both the specularly reflected wave component and the diffracted one. The breathing could be detected clearly in an amplitude versus time diagram. Its Doppler spectrum displayed a characteristic main/sideband component pattern, well-known for a phase-modulated sinusoidal signal. A relatively simple and fast MTI algorithm was tested to extract in real time the signal return from a moving metallic sphere in the shadow region behind the corner. The algorithm is based on coherent subtraction between different frequency sweeps of the 
stepped-frequency radar. Detection tracks could be produced in a time/down-range diagram upon IFFT of the resulting data.

The successful results of this paper, so far, encourage a continuation of the work, with tests in more realistic full-scale situations as a natural step toward practical implementation. Several problems have to be addressed in the future work. Perhaps, the foremost of these is the managing of the multipath situation, e.g., in the interpretation of data. However, this added complexity could be possibly used for positioning by combining multiple detections of a target, corresponding to different propagation paths, which requires that the geometry of the scattering environment be known.

\section{REFERENCES}

[1] M. Dehmollaian and K. Sarabandi, "Refocusing through building walls using Synthetic Aperture Radar," IEEE Trans. Geosci. Remote Sens., vol. 46, no. 6, pp. 1589-1599, Jun. 2008.

[2] C.-P. Lai, R. M. Narayanan, Q. Ruan, and A. Davydov, "Hilbert-Huang transform analysis of human activities using through-wall noise and noise-like radar," IET Radar Sonar Navig., vol. 2, no. 4, pp. 244-255, Aug. 2008.

[3] E. J. Baranoski, "Through-wall imaging: Historical perspective and future directions," J. Franklin Inst., vol. 345, no. 6, pp. 556-569, Sep. 2008.

[4] M. Farwell, J. Ross, R. Luttrell, D. Cohen, W. Chin, and T. Dogaru, "Sense through the wall system development and design considerations," J. Franklin Inst., vol. 345, no. 6, pp. 570-591, Sep. 2008.

[5] S. S. Ram, Y. Li, A. Lin, and H. Ling, "Doppler-based detection and tracking of humans in indoor environments," J. Franklin Inst., vol. 345, no. 6, pp. 679-699, Sep. 2008.

[6] T. Thayaparan, L. Stanković, and I. Djurović, "Micro-Doppler-based target detection and feature extraction in indoor and outdoor environments," J. Franklin Inst., vol. 345, no. 6, pp. 700-722, Sep. 2008.

[7] F. Soldovieri, R. Solimene, and R. Pierri, "A simple strategy to detect changes in through the wall imaging," Prog. Electromagn. Res. (PIER) $M$, vol. 7, pp. 1-13, 2009.

[8] Y.-S. Yoon and M. G. Amin, "Behind-the-wall target identification (BWTI)," Proc. SPIE, vol. 7308, pp. 73080S-1-73080S-12, 2009.

[9] N. Maaref, P. Millot, C. Pichot, and O. Picon, "A study of UWB FM-CW radar for the detection of human beings in motion inside a building," IEEE Trans. Geosci. Remote Sens., vol. 47, no. 5, pp. 1297-1300, May 2009.

[10] C. Debes, M. G. Amin, and A. M. Zoubir, "Target detection in singleand multiple-view through-the-wall radar imaging," IEEE Trans. Geosci. Remote Sens., vol. 47, no. 5, pp. 1349-1361, May 2009.

[11] E. Ertin and R. L. Moses, "Through-the-wall SAR attributed scattering center feature estimation," IEEE Trans. Geosci. Remote Sens., vol. 47, no. 5, pp. 1338-1348, May 2009.

[12] C. Le, T. Dogaru, L. Nguyen, and M. A. Ressler, "Ultrawideband (UWB) radar imaging of building interior: Measurements and predictions," IEEE Trans. Geosci. Remote Sens., vol. 47, no. 5, pp. 1409-1420, May 2009.

[13] A. Sume and A. Örbom, "See-around-corners with coherent radar. X-band measurements at Lilla Gåra, August 2007," Swedish Def. Res. Agency, Linköping, Sweden, Tech. Rep. FOI-R-2369-SE, Dec. 2007.

[14] A. Sume, M. Gustafsson, A. Jänis, S. Nilsson, and A. Örbom, "Seearound-corners with coherent radar," in Proc. 20th Nordic Conf. Radio Sci. Commun., RVK, Växjö, Sweden, Jun. 9-11, 2008, pp. 217-221.

[15] A. Sume, M. Gustafsson, A. Jänis, S. Nilsson, J. Rahm, and A. Örbom, "Radar detection of moving objects around corners," Proc. SPIE, vol. 7308, pp. 73080V-1-73080V-18, 2009.

[16] A. Sume, S. Nilsson, J. Rahm, and A. Örbom, "Radar measurements with realistic wall type for verification of the see-around-corners principle," Swedish Def. Res. Agency, Linköping, Sweden, Tech. Rep. FOI-R-2688SE, Dec. 2008

[17] J. L. Krolik, J. Farrell, and A. Steinhardt, "Exploiting multipath propagation for GMTI in urban environments," in Proc. IEEE Radar Conf., Verona, NY, Apr. 24-27, 2006, pp. 65-68.

[18] [Online]. Available: http://www.darpa.mil/sto/space/mer.html

[19] R. Linnehan and J. Schindler, "Multistatic scattering from moving targets in multipath environments," in Proc. IEEE Radar Conf., Pasadena, CA, May 4-8, 2009, pp. 1-6, Paper 3215.

[20] R. Linnehan and J. Schindler, "Validating multipath responses of moving targets through urban environments," in Proc. IEEE Int. Radar Conf., Washington, DC, May 10-14, 2010, pp. 1036-1041.
[21] P. Setlur, M. Amin, and F. Ahmad, "Multipath Doppler signatures from targets moving behind walls," in Proc. IEEE Int. Radar Conf., Washington, DC, May 10-14, 2010, pp. 799-803.

[22] M. D’Urso, F. Gianota, R. Lalli, and L. Infante, "Differential approach for through-the-wall life signs detection," in Proc. IEEE Int. Radar Conf., Washington, DC, May 10-14, 2010, pp. 1079-1082.

[23] F. He, G. Zhu, X. Huang, M. Mou, Z. Zhou, and C. Fan, "Preliminary results of ultra-wideband through-the-wall life-detecting radar," in Proc. IEEE Int. Radar Conf., Washington, DC, May 10-14, 2010, pp. 1327-1330.

[24] B. R. Mahafza, Introduction to Radar Analysis. Boca Raton, FL: CRC Press, 1998.

[25] D. A. McNamara, C. W. I. Pistorius, and J. A. G. Malherbe, Introduction to the Uniform Geometrical Theory of Diffraction. Boston, MA: Artech House, 1990.

[26] M. Gustafsson and S. Nilsson, "See around corners by use of radar," Swedish Def. Res. Agency, Linköping, Sweden, Sci. Rep. FOI-R-2324SE, Sep. 2007.

[27] F. Sagnard and G. El Zein, "In situ characterization of building materials for propagation modeling: Frequency and time responses," IEEE Trans. Antennas Propag., vol. 53, no. 10, pp. 3166-3173, Oct. 2005.

[28] A. Sume and T. Johansson, "Polarimetric wall penetrating radar-Theory, simulations, and state of development," Swedish Def. Res. Agency, Linköping, Sweden, Tech. Rep. FOI-R-2987-SE, Apr. 2010.

[29] A. Jänis, "Reflection properties of building wall materials and wall-like surfaces," Swedish Def. Res. Agency, Linköping, Sweden, Tech. Rep. FOI-R-2941-SE, Dec. 2009.

[30] S. Nilsson, A. Jänis, M. Gustafsson, J. Kjellgren, and A. Sume, "Through-the-wall high resolution imaging of a human and experimental characterization of the transmission of wall materials," Proc. SPIE, vol. 7117, pp. 71170L-1-71170L-16, 2008.

[31] M. Born and E. Wolf, Principles of Optics, 7th ed. Cambridge, U.K.: Cambridge Univ. Press, 1999.

[32] W. C. Stone, "Electromagnetic signal attenuation in construction materials," Nat. Inst. Stand. Technol. (NIST), Gaithersburg, MD, NIST Construction Automation Program, Rep. 3, NISTIR 6055, Oct. 1997.

[33] D. E. Barrick, "FM/CW radar signals and digital processing," Nat. Ocean. Atmos. Admin., Boulder, CO, Tech. Rep. ERL 283-WPL 26, Jul. 1973, NTIS AD-774 829.

[34] S. Qian and D. Chen, "Joint time-frequency analysis," IEEE Signal Process. Mag., vol. 16, no. 2, pp. 52-67, Mar. 1999.

[35] P. van Dorp and F. C. A. Groen, "Feature-based human motion parameter estimation with radar," IET Radar Sonar Navig., vol. 2, no. 2, pp. 135-145, Apr. 2008.

[36] J. E. Gray and S. R. Addison, "Effect of nonuniform target motion on radar backscattered waveforms," Proc. Inst. Elect. Eng.-Radar Sonar Navig., vol. 150, no. 4, pp. 262-270, Aug. 2003.

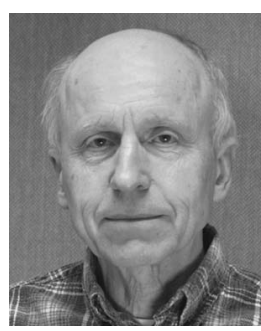

Ain Sume (M'84) received the M.Sc. degree in electrical engineering and the Ph.D. degree in space physics from the Chalmers University of Technology, Gothenburg, Sweden, in 1965 and 1978, respectively.

Between 1968 and 1978, he researched into interstellar microwave molecular lines at Onsala Space Observatory, Onsala, Sweden. Since 1978, he has been with the Swedish Defence Research Agency, Linköping, Sweden, involved with theoretical and experimental radar research including millimeterwave radar, target and background studies, polarization theory and application, and techniques for classification and recognition. His present activities are focused on radar applications in military and civil engagements in the urban scenario.

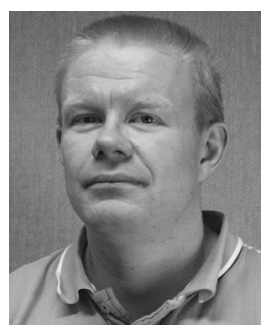

Magnus Gustafsson received the M.Sc. degree in applied physics and electrical engineering from Linköping University, Linköping, Sweden, in 2004. $\mathrm{He}$ is currently working toward the Ph.D. degree in the Department of Electrical and Information Technology, Lund University, Lund, Sweden.

$\mathrm{He}$ is currently with the Swedish Defence Research Agency, Linköping, where he joined in 2000 and where his activities include electromagnetic theory and computation. 


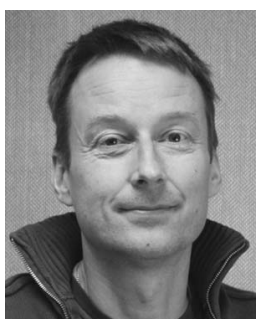

Magnus Herberthson received the M.Sc. degree in applied physics and electrical engineering and the Ph.D. degree in applied mathematics from Linköping University, Linköping, Sweden, in 1987 and 1993, respectively.

Since 1993, he has been with the Swedish Defence Research Agency (FOI), Linköping, Sweden, and with the Linköping University, where he became a Professor of applied mathematics in 2010. At FOI, his interests have been synthetic aperture radar, signal processing, and electromagnetic scattering theory. At Linköping University, his research field is the general theory of relativity.

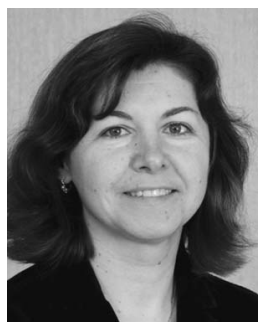

Anna Jänis received the M.Sc. degree in physics from Saint Petersburg State University, Saint Petersburg, Russia, in 1993. She is currently working toward the Ph.D. degree in materials technology at the Chalmers University of Technology, Gothenburg, Sweden, where her work covers the microwave absorbing properties of composites. In 2008, she received the Licentiate degree in materials technology concerning this work.

She is currently a Researcher with the Swedish Defence Research Agency (FOI), Linköping, Sweden, where she joined in 1998, working on radar absorbing materials.

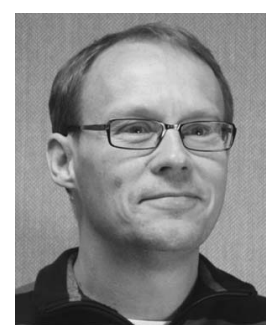

Stefan Nilsson received the B.Sc. degree in theoretical physics from Linköping University, Linköping, Sweden, in 1985.

$\mathrm{He}$ is currently with the Swedish Defence Research Agency (FOI), Linköping, where he joined in 1985 , to work with millimeter-wave radar technology. He is the Manager for projects concerning security sensors at FOI and is also the Leader of its test range for radar cross-section measurements. His current research interests include radar signatures and security sensors.

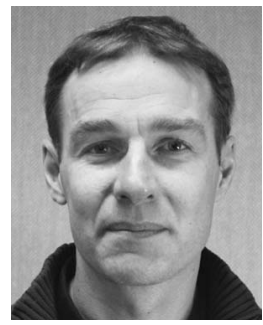

Jonas Rahm received the Ph.D. degree in nuclear physics from Uppsala University, Uppsala, Sweden, in 1997.

Since 1998, he has been with the Swedish Defence Research Agency, Linköping, Sweden, involved with theoretical and experimental radar research, signature management, and target recognition techniques. His present activities are focused on measurements and electromagnetic calculations on radar applications in military and civil engagements.

Anders Örbom, photograph and biography not available at the time of publication. 